\title{
Generalized Synchronization with Uncertain Parameters of Nonlinear Dynamic System via Adaptive Control
}

\author{
Cheng-Hsiung Yang and Cheng-Lin Wu \\ Graduate Institute of Automation and Control, National Taiwan University of Science and Technology, No. 43, Section 4, \\ Keelung Road, Taipei 106, Taiwan
}

Correspondence should be addressed to Cheng-Hsiung Yang; chyang123123@mail.ntust.edu.tw

Received 19 June 2014; Revised 18 August 2014; Accepted 18 August 2014; Published 11 September 2014

Academic Editor: M. Chadli

Copyright (C) 2014 C.-H. Yang and C.-L. Wu. This is an open access article distributed under the Creative Commons Attribution License, which permits unrestricted use, distribution, and reproduction in any medium, provided the original work is properly cited.

\begin{abstract}
An adaptive control scheme is developed to study the generalized adaptive chaos synchronization with uncertain chaotic parameters behavior between two identical chaotic dynamic systems. This generalized adaptive chaos synchronization controller is designed based on Lyapunov stability theory and an analytic expression of the adaptive controller with its update laws of uncertain chaotic parameters is shown. The generalized adaptive synchronization with uncertain parameters between two identical new LorenzStenflo systems is taken as three examples to show the effectiveness of the proposed method. The numerical simulations are shown to verify the results.
\end{abstract}

\section{Introduction}

The chaos synchronization phenomenon has the following feature: the trajectories of the master and the slave chaotic system are identical in spite of starting from different initial conditions or different nonlinear dynamic system. However, slight differentiations of initial conditions, for chaotic dynamical systems, will lead to completely different trajectories [1-14]. The issue may be treated as the control law design for observer of slave chaotic system using the master chaotic system so as to ensure that the controlled receiver synchronizes with the master chaotic system. Hence, the slave chaotic system completely traces the dynamics of the master chaotic system in the course of time [1519]. The key technique of chaos synchronization for secret communication has been widely investigated. Until now, a wide variety of approaches have been proposed for control and synchronization of chaotic systems, such as adaptive control [20, 21], backstepping control [22-25], sliding mode control [26-28], and fuzzy control [29-31], just to name a few. The forenamed strategies and many other existing skills of synchronization mainly concern the chaos synchronization of two identical chaotic systems with known parameters or identical unknown parameters [32-38].
Among many kinds of chaos synchronizations, the generalized synchronization is widely studied. This means that there exists a given functional relationship between the states of the master system and that of the slave system $y=f(x)$. In this paper, a new generalized synchronization with uncertain parameters,

$$
\begin{aligned}
& \dot{x}=f(t, x, A(t)), \\
& y=x+F(t),
\end{aligned}
$$

is studied, where $x, y$ are the state vectors of the master and slave system, respectively, and the $A(t)$ is uncertain chaotic parameters in $f$. The $F(t)$ may be given a regular/chaotic dynamical system.

The rest of the paper is organized as follows. In Section 2, by the Lyapunov asymptotic stability theorem, the generalized synchronization with uncertain chaotic parameters by adaptive control scheme is given. In Section 3, various adaptive controllers and update laws are designed for the generalized synchronization with uncertain parameters of the identical Lorenz-Stenflo systems. The numerical simulation of three examples is also given in Section 3. Finally, some concluding remarks are given in Section 4. 


\section{Generalized Adaptive Synchronization with Uncertain Parameters Scheme}

Consider the master system

$$
\dot{x}=f(t, x, A(t))
$$

and the slave system

$$
\dot{y}=f(t, y, \widehat{A}(t))+u,
$$

where $x=\left[x_{1}, x_{2}, \ldots, x_{n}\right]^{T} \in R^{n}, y=\left[y_{1}, y_{2}, \ldots, y_{n}\right]^{T} \in R^{n}$ denote the master states vector and slave states vector, respectively, the $f$ is nonlinear vector functions, the $A(t)$ is uncertain chaotic parameters in $f$, the $\widehat{A}(t)$ is estimates of uncertain chaotic parameters in $f$, and the $u=\left[u_{1}, u_{2}, \ldots, u_{n}\right]^{T} \in R^{n}$ is adaptive control vector.

Our goal is to design a controller $u(t)$ and an adaptive law $\dot{\widetilde{A}}$ so that the state vector of the slave system equation (3) asymptotically approaches the state vector of the master system equation (2) plus a given vector regular/chaotic function $F(t)=\left[F_{1}(t), F_{2}(t), \ldots, F_{n}(t)\right]^{T}$, and finally the generalized adaptive synchronization with uncertain parameters will be accomplished in the sense that the limit of the states error vector $e(t)=\left[e_{1}, e_{2}, \ldots, e_{n}\right]^{T}$ and parameters error vector $\widetilde{A}(t)=\left[\widetilde{A}_{1}, \widetilde{A}_{2}, \ldots, \widetilde{A}_{m}\right]^{T}$ approaches zero:

$$
\begin{aligned}
& \lim _{t \rightarrow \infty} e(t)=0, \\
& \lim _{t \rightarrow \infty} \widetilde{A}(t)=0,
\end{aligned}
$$

where $e_{i}=x_{i}-y_{i}+F_{i}(t),(i=1,2, \ldots, n)$ and $\widetilde{A}_{j}=A_{j}(t)-$ $\widehat{A}_{j}(t),(j=1,2, \ldots, m)$.

From (4a), we have

$$
\dot{e}_{i}=\dot{x}_{i}-\dot{y}_{i}+\dot{F}_{i}(t), \quad i=1,2, \ldots, n .
$$

Introduce (2) and (3) in (5) as

$$
\dot{e}=f(t, x, A(t))-f(t, y, \widehat{A}(t))+\dot{F}(t)-u(t) .
$$

A Lyapunov function candidate $V(e, \widetilde{A})$ is chosen as a positive definite function as

$$
V(e, \widetilde{A})=\frac{1}{2} e^{T} e+\frac{1}{2} \widetilde{A}^{T} \widetilde{A} .
$$

Its derivative along the solution of (7) is

$$
\begin{aligned}
\dot{V}(e, \widetilde{A})= & e^{T}[f(t, x, A(t))-f(t, y, \widehat{A}(t))+\dot{F}(t)-u(t)] \\
+ & \widetilde{A}^{T} \dot{\widetilde{A}}
\end{aligned}
$$

where $u(t)$ and $\dot{\widetilde{A}}$ are chosen so that $\dot{V}=e^{T} C_{1} e+\widetilde{A}^{T} C_{2} \widetilde{A}$, $C_{1}$ and $C_{2}$ are negative constants, and $\dot{V}$ is a negative definite function of $e_{1}, e_{2}, \ldots, e_{n}$ and $\widetilde{A}_{1}, \widetilde{A}_{2}, \ldots, \widetilde{A}_{m}$. When

$$
\lim _{t \rightarrow \infty} e=0, \quad \lim _{t \rightarrow \infty} \widetilde{A}=0,
$$

the generalized adaptive synchronization with uncertain parameters is obtained.

\section{Results of Numerical Simulation}

In this section, a mathematical proof is provided for the three cases' results of numerical, adaptive synchronization, generalized adaptive synchronization, and generalized adaptive synchronization with uncertain parameters.

3.1. Case I Adaptive Synchronization. The master system is new Lorenz-Stenflo system [39]:

$$
\begin{aligned}
& \dot{x}_{1}=a\left(x_{2}-x_{1}\right), \\
& \dot{x}_{2}=c x_{1}-x_{1} x_{3}-x_{2}, \\
& \dot{x}_{3}=b x_{4}-d x_{3}+x_{1} x_{2}, \\
& \dot{x}_{4}=-x_{1}-a x_{4},
\end{aligned}
$$

where $a=3.7, b=1.5, c=26$, and $d=0.7$. The initial conditions are $x_{1}(0)=30, x_{2}(0)=30, x_{3}(0)=30$, and $x_{4}(0)=30$. The phase portrait is shown in Figure 1 .

The slave system is

$$
\begin{aligned}
& \dot{y}_{1}=\widehat{a} y_{2}-\widehat{a} y_{1}+u_{1}, \\
& \dot{y}_{2}=\widehat{c} y_{1}-y_{1} y_{3}-y_{2}+u_{2}, \\
& \dot{y}_{3}=y_{1} y_{2}-\widehat{d} y_{3}+\widehat{b} y_{4}+u_{3}, \\
& \dot{y}_{4}=-y_{1}-\widehat{a} y_{4}+u_{4},
\end{aligned}
$$

where $\widehat{a}, \widehat{b}, \widehat{c}, \widehat{d}$, and $\widehat{r}$ are estimates of uncertain parameters $a, b, c, d$, and $r$, respectively. The initial conditions of salve system are $y_{1}(0)=-50, y_{2}(0)=-50, y_{3}(0)=-50$, and $y_{4}(0)=-50$.

Our objective is to design the controllers such that the trajectories, $x(t)$ and $y(t)$, of the master system and slave system satisfy

$$
\lim _{t \rightarrow \infty}\|x(t)-y(t)\|=0 \text {. }
$$

Our objective is to design the controllers parameters estimation update laws $\dot{\widetilde{A}}=[\dot{\tilde{a}}, \dot{\widetilde{b}}, \dot{\widetilde{c}}, \dot{\tilde{d}}]^{T}$ such that the trajectories, $A(t)$ and $\widehat{A}(t)$, of the uncertain chaotic parameters and estimates of uncertain chaotic parameters satisfy

$$
\lim _{t \rightarrow \infty}\|A(t)-\widehat{A}(t)\|=0,
$$

where $\|\cdot\|$ denotes the Euclidean norm.

Define an error vector function

$$
e_{i}=x_{i}-y_{i}, \quad(i=1,2,3,4) .
$$






(a)



(b)

FIgURE 1: Three-dimension phase portrait of the four-dimensional Lorenz-Stenflo system and its projection.

From the error functions, we get the error dynamics

$$
\begin{aligned}
& \dot{e}_{1}=\widehat{a} e_{2}+\widetilde{a} x_{2}-\widehat{a} e_{1}-\tilde{a} x_{1}-u_{1}, \\
& \dot{e}_{2}=\widehat{c} e_{1}+\widetilde{c} x_{1}+e_{1} e_{3}-x_{1} e_{3}-x_{3} e_{1}-e_{2}-u_{2}, \\
& \dot{e}_{3}=-e_{1} e_{2}+x_{1} e_{2}+x_{2} e_{1}-\widehat{d} e_{3}-\tilde{d} x_{3}+\widehat{b} e_{4}+\widetilde{b} x_{4}-u_{3}, \\
& \dot{e}_{4}=-e_{1}-\widehat{a} e_{4}-\tilde{a} x_{4}-u_{4},
\end{aligned}
$$

where $e_{1}=x_{1}-y_{1}, e_{2}=x_{2}-y_{2}, e_{3}=x_{3}-y_{3}, e_{4}=x_{4}-y_{4}$, $\widetilde{a}=a-\widehat{a}, \widetilde{b}=b-\widehat{b}, \widetilde{c}=c-\widehat{c}$, and $\widetilde{d}=d-\widehat{d}$.

Choose a Lyapunov function candidate in the form of a positive definite function

$$
\begin{aligned}
& V\left(e_{1}, e_{2}, e_{3}, e_{4}, \tilde{a}, \tilde{b}, \tilde{c}, \widetilde{d}\right) \\
& \quad=\frac{1}{2}\left(e_{1}^{2}+e_{2}^{2}+e_{3}^{2}+e_{4}^{2}+\widetilde{a}^{2}+\widetilde{b}^{2}+\widetilde{c}^{2}+\widetilde{d}^{2}\right),
\end{aligned}
$$

and its time derivative is

$$
\begin{aligned}
& \dot{V}\left(e_{1}, e_{2}, e_{3}, e_{4}, \tilde{a}, \tilde{b}, \widetilde{c}, \widetilde{d}\right) \\
& =e_{1} \dot{e}_{1}+e_{2} \dot{e}_{2}+e_{3} \dot{e}_{3}+e_{4} \dot{e}_{4}+\tilde{a} \dot{\tilde{a}}+\tilde{b} \dot{\tilde{b}}+\tilde{\tilde{c}}+\tilde{\tilde{d}} \\
& =e_{1}\left(\widehat{a} e_{2}+\widetilde{a} x_{2}-\widehat{a} e_{1}-\tilde{a} x_{1}-u_{1}\right) \\
& \quad+e_{2}\left(\widehat{c} e_{1}+\widetilde{c} x_{1}+e_{1} e_{3}-x_{1} e_{3}-x_{3} e_{1}-e_{2}-u_{2}\right) \\
& +e_{3}\left(-e_{1} e_{2}+x_{1} e_{2}+x_{2} e_{1}-\widehat{d} e_{3}-\tilde{d} x_{3}\right. \\
& \left.\quad+\widehat{b} e_{4}+\tilde{b} x_{4}-u_{3}\right)
\end{aligned}
$$

$$
\begin{aligned}
& +e_{4}\left(-e_{1}-\widehat{a} e_{4}-\tilde{a} x_{4}-u_{4}\right)+\widetilde{a}(-\dot{\hat{a}})+\widetilde{b}(-\dot{\hat{b}}) \\
& +\widetilde{c}(-\dot{\hat{c}})+\widetilde{d}(-\dot{\vec{d}})+\widetilde{r}(-\dot{\hat{r}}) .
\end{aligned}
$$

Choose the parameters estimation update laws as follows:

$$
\begin{aligned}
& \dot{\tilde{a}}=-\dot{\vec{a}}=-e_{1}\left(x_{2}-x_{1}\right)+e_{4} x_{4}-\tilde{a}, \\
& \dot{\vec{b}}=-\dot{\vec{b}}=-e_{3} x_{4}-\tilde{b}, \\
& \dot{\tilde{c}}=-\dot{\widehat{c}}=-e_{2} x_{1}-\tilde{c}, \\
& \dot{\vec{d}}=-\dot{\vec{d}}=e_{3} x_{3}-\tilde{d} .
\end{aligned}
$$

The initial values of estimates for uncertain parameters are $\widehat{a}(0)=0, \widehat{b}(0)=0, \widehat{c}(0)=0$, and $\widehat{d}(0)=0$. Through (16) and (17), the appropriate controllers can be designed as

$$
\begin{aligned}
& u_{1}=\widehat{a} e_{2}-\widehat{a} e_{1}+e_{1}, \\
& u_{2}=\widehat{c} e_{1}+e_{1} e_{3}-x_{1} e_{3}-x_{3} e_{1}-e_{2}+e_{2}, \\
& u_{3}=-e_{1} e_{2}+x_{1} e_{2}+x_{2} e_{1}-\widehat{d} e_{3}+\widehat{b} e_{4}+e_{3}, \\
& u_{4}=-e_{1}-\widehat{a} e_{4}+e_{4} .
\end{aligned}
$$

Substituting (18) and (17) into (16), we obtain

$$
\begin{aligned}
& \dot{V}\left(e_{1}, e_{2}, e_{3}, e_{4}, \tilde{a}, \tilde{b}, \widetilde{c}, \widetilde{d}\right) \\
& \quad=-e_{1}{ }^{2}-e_{2}{ }^{2}-e_{3}{ }^{2}-e_{4}{ }^{2}-\widetilde{a}^{2}-\widetilde{b}^{2}-\widetilde{c}^{2}-\widetilde{d}^{2}<0 .
\end{aligned}
$$

Since the Lyapunov function $V\left(e_{1}, e_{2}, e_{3}, e_{4}, \widetilde{a}, \widetilde{b}, \widetilde{c}, \widetilde{d}\right)$ is positive definite and its derivative $\dot{V}\left(e_{1}, e_{2}, e_{3}, e_{4}, \tilde{a}, \widetilde{b}, \widetilde{c}, \widetilde{d}\right)$ is 

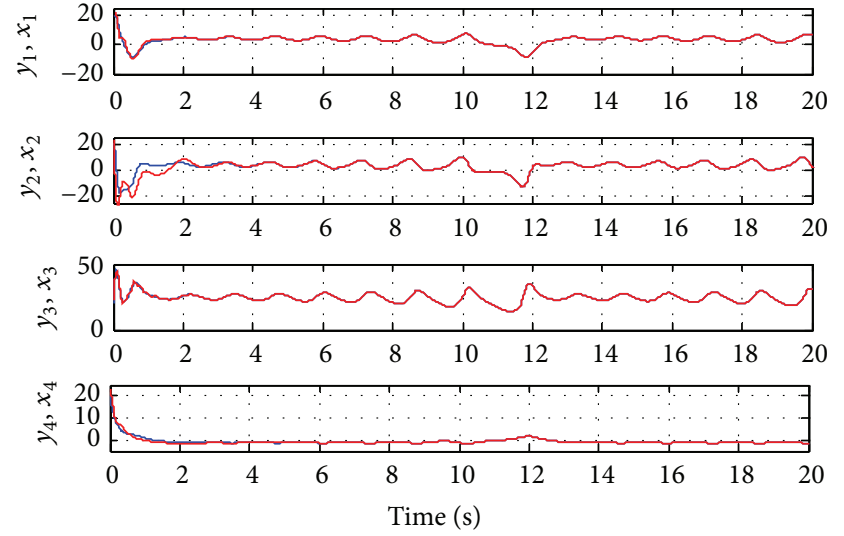

Master
- Slave

FIGURE 2: Time histories of the master system and slave system for Case I.
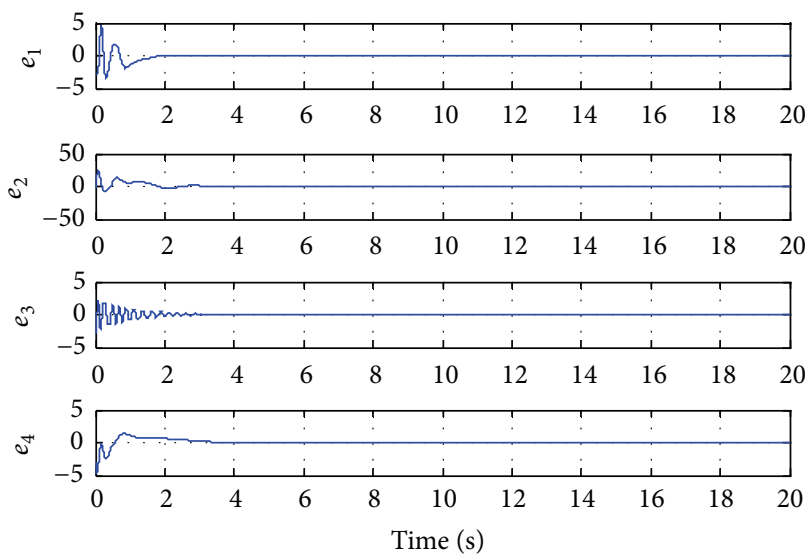

FIGURE 3: Time histories of error states for Case I.

negative definite in the neighborhood of the zero solutions for (12a) and (12b), according to the Lyapunov stability theory, the zero solutions of error states dynamic and parameters error vector are asymptotically stable; namely, the slave system equation (11) can asymptotically converge to its master system equation (10) with the adaptive control law equation (18) and the estimation parameter update law equation (17). The adaptive synchronization concept proof had to be completed. The numerical simulation results are shown in Figures 2, 3, and 4.

3.2. Case II Generalized Adaptive Synchronization. The given functional system for generalized synchronization is also a new Lorenz-Stenflo system but with different initial conditions: $w_{1}(0)=25, w_{2}(0)=25, w_{3}(0)=25$, and $w_{4}(0)=25$ :

$$
\begin{aligned}
& \dot{w}_{1}=a\left(w_{2}-w_{1}\right), \\
& \dot{w}_{2}=c w_{1}-w_{1} w_{3}-w_{2},
\end{aligned}
$$
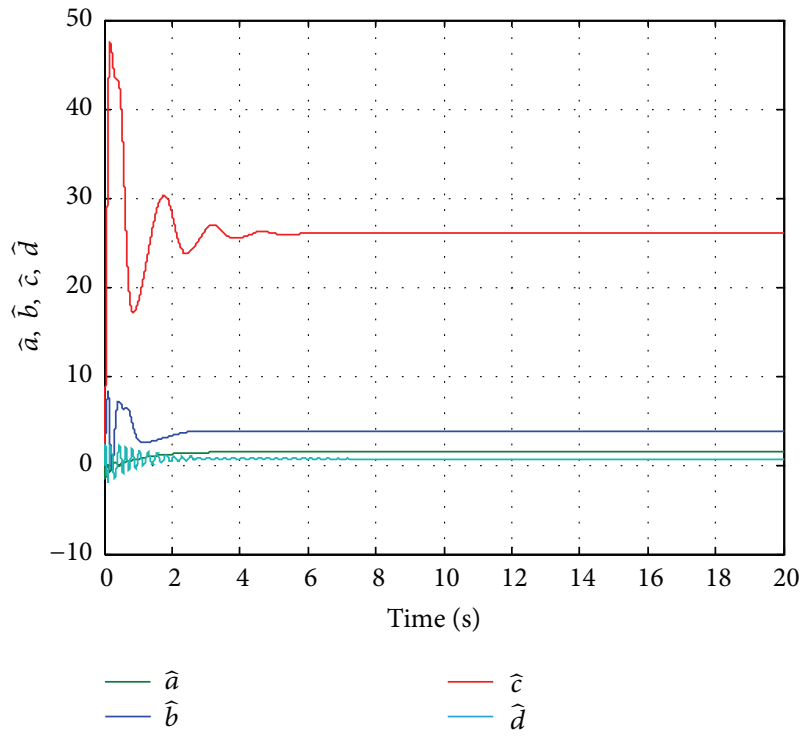

FIGURE 4: Time histories of estimated parameters for Case I.

$$
\begin{aligned}
& \dot{w}_{3}=b w_{4}-d w_{3}+w_{1} w_{2}, \\
& \dot{w}_{4}=-a w_{4}-w_{1} .
\end{aligned}
$$

When the time approaches infinite, the error functions approach zero. The generalized adaptive synchronization can be accomplished as

$$
\lim _{t \rightarrow \infty} e=0,
$$

where the error functions here can be defined as

$$
e_{i}=x_{i}+w_{i}-y_{i}, \quad(i=1,2,3,4)
$$

From the error functions equation (22), we get the error dynamics

$$
\begin{aligned}
\dot{e}_{1}= & \widehat{a}\left(e_{2}-e_{1}\right)+\widetilde{a}\left(x_{2}-x_{1}+w_{2}-w_{1}\right)-u_{1}, \\
\dot{e}_{2}= & \widehat{c} e_{1}+\tilde{c}\left(x_{1}+w_{1}\right)-e_{2}+x_{1}\left(w_{3}-e_{3}\right)+w_{1}\left(x_{3}-e_{3}\right) \\
& -e_{1}\left(x_{3}+w_{3}-e_{3}\right)-u_{2}, \\
\dot{e}_{3}= & -\widehat{d} e_{3}-\widetilde{d}\left(x_{3}+w_{3}\right)+\widehat{b} e_{4}+\widetilde{b}\left(x_{4}+w_{4}\right) \\
& -x_{1}\left(w_{2}-e_{2}\right)-w_{1}\left(x_{2}-e_{2}\right)+\left(x_{2}+w_{2}-e_{2}\right)-u_{3}, \\
\dot{e}_{4}= & -e_{1}-\widehat{a} e_{4}-\tilde{a}\left(x_{4}+w_{4}\right)-u_{4},
\end{aligned}
$$

where $\tilde{a}=a-\widehat{a}, \widetilde{b}=b-\widehat{b}, \widetilde{c}=c-\widehat{c}$, and $\widetilde{d}=d-\widehat{d}$. 
Choose a Lyapunov function in the form of a positive definite function

$$
\begin{aligned}
V & \left(e_{1}, e_{2}, e_{3}, e_{4}, \tilde{a}, \widetilde{b}, \widetilde{c}, \widetilde{d}\right) \\
& =\frac{1}{2}\left(e_{1}^{2}+e_{2}^{2}+e_{3}^{2}+e_{4}^{2}+\widetilde{a}^{2}+\widetilde{b}^{2}+\widetilde{c}^{2}+\widetilde{d}^{2}\right),
\end{aligned}
$$

and its time derivative is

$$
\begin{aligned}
& \dot{V}\left(e_{1}, e_{2}, e_{3}, e_{4}, \tilde{a}, \tilde{b}, \tilde{c}, \tilde{d}\right) \\
& =e_{1} \dot{e}_{1}+e_{2} \dot{e}_{2}+e_{3} \dot{e}_{3}+e_{4} \dot{e}_{4}+\tilde{a} \dot{a}+\tilde{a} \dot{\widetilde{b}}+\dot{\overrightarrow{c c}}+\dot{\widetilde{d d}} \\
& =e_{1}\left(\widehat{a}\left(e_{2}-e_{1}\right)+\widetilde{a}\left(x_{2}-x_{1}+w_{2}-w_{1}\right)-u_{1}\right) \\
& +e_{2}\left(\widehat{c} e_{1}+\tilde{c}\left(x_{1}+w_{1}\right)-e_{2}+x_{1}\left(w_{3}-e_{3}\right)\right. \\
& \left.+w_{1}\left(x_{3}-e_{3}\right)-e_{1}\left(x_{3}+w_{3}-e_{3}\right)-u_{2}\right) \\
& +e_{3}\left(-\widehat{d} e_{3}-\tilde{d}\left(x_{3}+w_{3}\right)+\hat{b} e_{4}+\widetilde{b}\left(x_{4}+w_{4}\right)\right. \\
& -x_{1}\left(w_{2}-e_{2}\right)-w_{1}\left(x_{2}-e_{2}\right) \\
& \left.+e_{1}\left(x_{2}+w_{2}-e_{2}\right)-u_{3}\right) \\
& +e_{4}\left(-e_{1}-\widehat{a} e_{4}-\widetilde{a}\left(x_{4}+w_{4}\right)-u_{4}\right)+\widetilde{a}(-\dot{\hat{a}})+\widetilde{b}(-\dot{\vec{b}}) \\
& +\widetilde{c}(-\dot{\vec{c}})+\widetilde{d}(-\dot{\vec{d}})+\widetilde{r}(-\dot{\vec{r}}) .
\end{aligned}
$$

Choose the parameters estimation update laws as follows:

$$
\begin{aligned}
& \dot{\tilde{a}}=-\dot{\vec{a}}=-e_{1}\left(x_{2}-x_{1}+w_{2}-w_{1}\right)+e_{4}\left(x_{4}+w_{4}\right)-\tilde{a}, \\
& \dot{\vec{b}}=-\dot{\vec{b}}=-e_{3}\left(x_{4}+w_{4}\right)-\widetilde{b}, \\
& \dot{\tilde{c}}=-\dot{\vec{c}}=-e_{2}\left(x_{1}+w_{1}\right)-\widetilde{c}, \\
& \dot{\tilde{d}}=-\dot{\vec{d}}=e_{3}\left(x_{3}+w_{3}\right)-\tilde{d} .
\end{aligned}
$$

The initial values of estimates for uncertain parameters are $\widehat{a}(0)=0, \widehat{b}(0)=0, \widehat{c}(0)=0$, and $\widehat{d}(0)=0$. Through $(25)$ and (26), the appropriate controllers can be designed as

$$
\begin{aligned}
u_{1}= & \widehat{a} e_{2}-\widehat{a} e_{1}+e_{1}, \\
u_{2}= & \widehat{c} e_{1}-e_{2}+x_{1}\left(w_{3}-e_{3}\right)+w_{1}\left(x_{3}-e_{3}\right) \\
& -e_{1}\left(x_{3}+w_{3}-e_{3}\right)+e_{2}, \\
u_{3}= & -\widehat{d} e_{3}+\widehat{b} e_{4}-x_{1}\left(w_{2}-e_{2}\right)-w_{1}\left(x_{2}-w_{2}\right) \\
& +e_{1}\left(x_{2}+w_{2}-e_{2}\right)+e_{3}, \\
u_{4}= & -e_{1}-\widehat{a} e_{4}+e_{4} .
\end{aligned}
$$

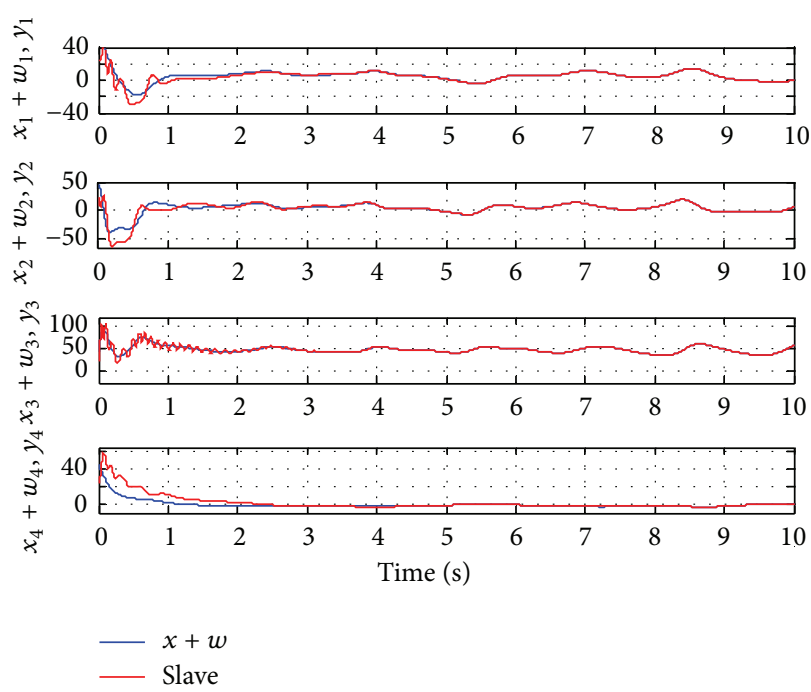

FIgURE 5: Time histories of the generalized signal (master $x$ system plus given function $w$ ) and slave system for Case II.
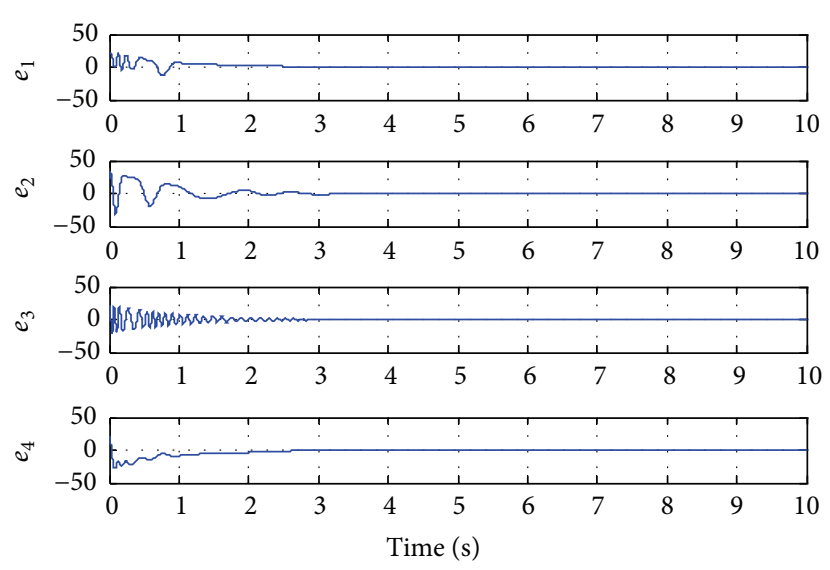

FIGURE 6: Time histories of error states for Case II.

Substituting (26) and (27) into (25), we obtain

$$
\begin{aligned}
& \dot{V}\left(e_{1}, e_{2}, e_{3}, e_{4}, \tilde{a}, \widetilde{b}, \widetilde{c}, \widetilde{d}\right) \\
& \quad=-e_{1}{ }^{2}-e_{2}{ }^{2}-e_{3}{ }^{2}-e_{4}{ }^{2}-\widetilde{a}^{2}-\widetilde{b}^{2}-\widetilde{c}^{2}-\widetilde{d}^{2}<0 .
\end{aligned}
$$

Since the Lyapunov function $V\left(e_{1}, e_{2}, e_{3}, e_{4}, \widetilde{a}, \widetilde{b}, \widetilde{c}, \widetilde{d}\right)$ is positive definite and its derivative $\dot{V}\left(e_{1}, e_{2}, e_{3}, e_{4}, \widetilde{a}, \widetilde{b}, \widetilde{c}, \widetilde{d}\right)$ is negative definite in the neighborhood of the zero solutions for (12a) and (12b), according to the Lyapunov stability theory, the zero solutions of error states dynamic and parameters error vector are asymptotically stable; namely, the slave system equation (11) can asymptotically converge to its master system equation (10) with the adaptive control law equation (27) and the estimation parameter update law equation (26). The generalized adaptive synchronization concept proof had to be completed. The numerical simulation results are shown in Figures 5, 6, and 7. 


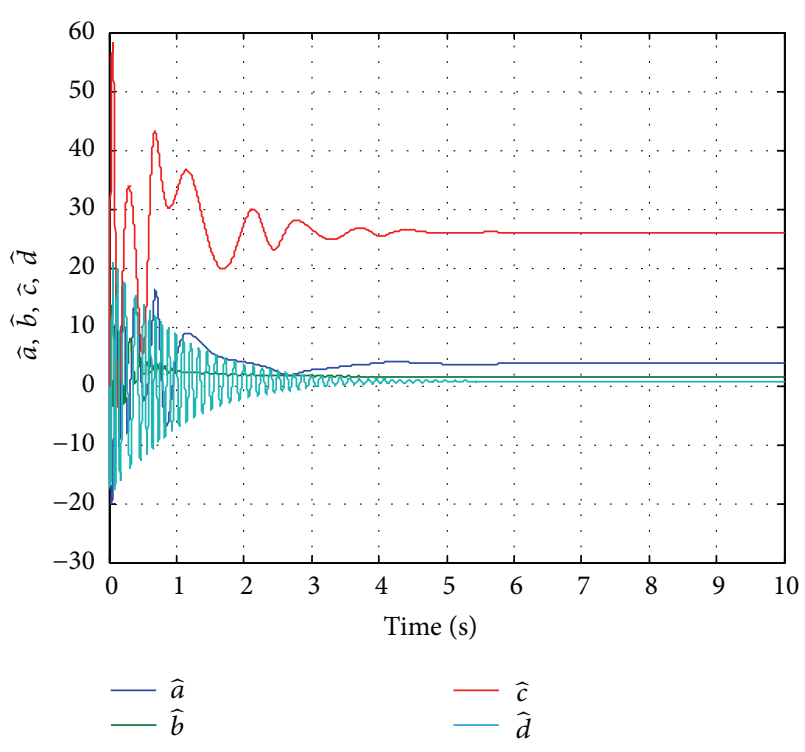

FIGURE 7: Time histories of estimated parameters for Case II.

3.3. Case III Generalized Adaptive Synchronization with Uncertain Parameters. Consider that the master system is the new Lorenz-Stenflo system with uncertain chaotic parameters

$$
\begin{aligned}
& \dot{x}_{1}=A_{1}(t)\left(x_{2}-x_{1}\right), \\
& \dot{x}_{2}=A_{3}(t) x_{1}-x_{1} x_{3}-x_{2}, \\
& \dot{x}_{3}=A_{2}(t) x_{3}-A_{4}(t) x_{3}+x_{1} x_{2}, \\
& \dot{x}_{4}=-A_{1}(t) x_{4}-x_{1},
\end{aligned}
$$

where $A_{1}(t), A_{2}(t), A_{3}(t)$, and $A_{4}(t)$ are uncertain chaotic parameters. The uncertain parameters are given as

$$
\begin{aligned}
& A_{1}(t)=a\left(1+f_{1} z_{1}\right), \\
& A_{2}(t)=b\left(1+f_{2} z_{2}\right), \\
& A_{3}(t)=c\left(1+f_{3} z_{3}\right), \\
& A_{4}(t)=d\left(1+f_{4} z_{4}\right),
\end{aligned}
$$

where $f_{1}, f_{2}, f_{3}$, and $f_{4}$ are arbitrary positive constants. Positive constants are $f_{1}=f_{2}=f_{3}=f_{4}=0.005$. The chaotic signals $z_{1}, z_{2}, z_{3}$, and $z_{4}$ are given as the states of system as follows:

$$
\begin{aligned}
& \dot{z}_{1}=a\left(z_{2}-z_{1}\right), \\
& \dot{z}_{2}=c z_{1}-z_{1} z_{3}-z_{2}, \\
& \dot{z}_{3}=b z_{4}+-d z_{3}+z_{1} z_{2}, \\
& \dot{z}_{4}=-a z_{4}-z_{1} .
\end{aligned}
$$

The initial constants of the chaotic signals are $z_{1}(0)=0.7$, $z_{2}(0)=0.7, z_{3}(0)=0.7$, and $z_{4}(0)=0.7$. The new LorenzStenflo system with uncertain chaotic parameters of master system will exhibit a more complex dynamic behavior since the parameters of the system change over time.

The generalized synchronization error functions can be defined as

$$
e_{i}=x_{i}+w_{i}-y_{i}, \quad(i=1,2,3,4)
$$

From the error functions equation (32), the error dynamics becomes

$$
\begin{aligned}
\dot{e}_{1}= & \widehat{A}_{1}\left(e_{2}-e_{1}-w_{2}+w_{1}\right)+\widetilde{A}_{1}\left(x_{2}-x_{1}\right) \\
& +a\left(w_{2}-w_{1}\right)-u_{1}, \\
\dot{e}_{2}= & \widehat{A}_{3}\left(e_{1}-w_{1}\right)+\widetilde{A}_{3} x_{1}+c w_{1}+x_{1}\left(w_{3}-e_{3}\right) \\
& +w_{1}\left(x_{3}-e_{3}\right)-e_{1}\left(x_{3}+w_{3}-e_{3}\right)-e_{2}-u_{2}, \\
\dot{e}_{3}= & \widehat{A}_{2}\left(e_{4}-w_{4}\right)+\widetilde{A}_{2} x_{4}+b w_{4}-\widehat{A}_{4}\left(e_{3}-w_{3}\right)-\widetilde{A}_{4} x_{3} \\
& -d w_{3}-x_{1}\left(w_{2}-e_{2}\right)-w_{1}\left(x_{2}-e_{2}\right) \\
& +e_{1}\left(x_{2}+w_{2}-e_{2}\right)-u_{3}, \\
\dot{e}_{4}= & -e_{1}-\widehat{A}_{1}\left(e_{4}-w_{4}\right)-\widetilde{A}_{1} x_{4}-a w_{4}-u_{4},
\end{aligned}
$$

where $\widetilde{A}_{i}(t)=A_{i}(t)-\widehat{A}_{i}(t)(i=1,2,3,4)$.

Choose a Lyapunov function in the form of a positive definite function:

$$
\begin{aligned}
& V\left(e_{1}, e_{2}, e_{3}, e_{4}, \widetilde{A}_{1}, \widetilde{A}_{2}, \widetilde{A}_{3}, \widetilde{A}_{4}\right) \\
& =\frac{1}{2}\left(e_{1}^{2}+e_{2}^{2}+e_{3}^{2}+e_{4}^{2}+\widetilde{A}_{1}^{2}(t)+\widetilde{A}_{2}^{2}(t)\right. \\
& \left.\quad+\widetilde{A}_{3}^{2}(t)+\widetilde{A}_{4}^{2}(t)\right),
\end{aligned}
$$

and its time derivative is

$$
\begin{aligned}
& \dot{V}\left(e_{1}, e_{2}, e_{3}, e_{4}, \widetilde{A}_{1}, \widetilde{A}_{2}, \widetilde{A}_{3}, \widetilde{A}_{4}\right) \\
& =e_{1} \dot{e}_{1}+e_{2} \dot{e}_{2}+e_{3} \dot{e}_{3}+e_{4} \dot{e}_{4}+\widetilde{A}_{1} \dot{\vec{A}}_{1}+\widetilde{A}_{2} \dot{\vec{A}}_{2} \\
& +\widetilde{A}_{3} \dot{\widetilde{A}}_{3}+\widetilde{A}_{4} \dot{\widetilde{A}}_{4} \\
& =e_{1}\left(\widehat{A}_{1}\left(e_{2}-e_{1}-w_{2}+w_{1}\right)+\widetilde{A}_{1}\left(x_{2}-x_{1}\right)\right. \\
& \left.+a\left(w_{2}-w_{1}\right)-u_{1}\right) \\
& +e_{2}\left(\widehat{A}_{3}\left(e_{1}-w_{1}\right)+\widetilde{A}_{3} x_{1}+c w_{1}+x_{1}\left(w_{3}-e_{3}\right)\right. \\
& \left.\quad+w_{1}\left(x_{3}-e_{3}\right)-e_{1}\left(x_{3}+w_{3}-e_{3}\right)-e_{2}-u_{2}\right) \\
& +e_{3}\left(\widehat{A}_{2}\left(e_{4}-w_{4}\right)+\widetilde{A}_{2} x_{4}+b w_{4}-\widehat{A}_{4}\left(e_{3}-w_{3}\right)\right. \\
& \quad-\widetilde{A}_{4} x_{3}-d w_{3}-x_{1}\left(w_{2}-e_{2}\right)-w_{1}\left(x_{2}-e_{2}\right) \\
& \left.\quad+e_{1}\left(x_{2}+w_{2}-e_{2}\right)-u_{3}\right)
\end{aligned}
$$




$$
\begin{aligned}
& +e_{4}\left(-e_{1}-\widehat{A}_{1}\left(e_{4}-w_{4}\right)-\widetilde{A}_{1} x_{4}-a w_{4}-u_{4}\right) \\
& +\widetilde{A}_{1}\left(-\dot{\widehat{A}}_{1}\right)+\widetilde{A}_{2}\left(-\dot{\widehat{A}}_{2}\right)+\widetilde{A}_{3}\left(-\dot{\widehat{A}}_{3}\right)+\widetilde{A}_{4}\left(-\dot{\widehat{A}}_{4}\right) .
\end{aligned}
$$

Choose the parameters estimation update laws for those uncertain parameters as follows:

$$
\begin{aligned}
& \dot{\widetilde{A}}_{1}=\dot{A}_{1}-\dot{\widehat{A}}_{1}=-e_{1}\left(x_{2}-x_{1}\right)+e_{4} x_{4}-\widetilde{A}_{1}, \\
& \dot{\widetilde{A}}_{2}=\dot{A}_{2}-\dot{\widehat{A}}_{2}=-e_{3} x_{4}-\widetilde{A}_{2}, \\
& \dot{\bar{A}}_{3}=\dot{A}_{3}-\dot{A}_{3}=-e_{2} x_{1}-\widetilde{A}_{3}, \\
& \dot{\vec{A}}_{4}=\dot{A}_{4}-\dot{\bar{A}}_{4}=e_{3} x_{3}-\widetilde{A}_{4} .
\end{aligned}
$$

The initial values of estimates for uncertain parameters are $\widehat{A}_{1}(0)=0, \widehat{A}_{2}(0)=0, \widehat{A}_{3}(0)=0$, and $\widehat{A}_{4}(0)=0$. Through (35) and (36), the appropriate controllers can be designed as

$$
\begin{aligned}
u_{1}= & \widehat{A}_{1}\left(e_{2}-e_{1}-w_{2}+w_{1}\right)+a\left(w_{2}-w_{1}\right)+e_{1}, \\
u_{2}= & \widehat{A}_{3}\left(e_{1}-w_{1}\right)+c w_{1}+x_{1}\left(w_{3}-e_{3}\right)+w_{1}\left(x_{3}-e_{3}\right) \\
& -e_{1}\left(x_{3}+w_{3}-e_{3}\right)-e_{2}+e_{2}, \\
u_{3}= & \widehat{A}_{2}\left(e_{4}-w_{4}\right)+b w_{4}-\widehat{A}_{4}\left(e_{3}-w_{3}\right)-d w_{3} \\
& -x_{1}\left(w_{2}-e_{2}\right)-w_{1}\left(x_{2}-w_{2}\right) \\
& +e_{1}\left(x_{2}+w_{2}-e_{2}\right)+e_{3}, \\
u_{4}= & -\widehat{A}_{1}\left(e_{4}-w_{4}\right)-a w_{4}-e_{1}+e_{4} .
\end{aligned}
$$

Substituting (36) and (37) into (35), we obtain

$$
\begin{aligned}
\dot{V} & \left(e_{1}, e_{2}, e_{3}, e_{4}, \widetilde{A}_{1}, \widetilde{A}_{2}, \widetilde{A}_{3}, \widetilde{A}_{4}\right) \\
& =-e_{1}^{2}-e_{2}^{2}-e_{3}^{2}-e_{4}^{2}-\widetilde{A}_{1}^{2}(t)-\widetilde{A}_{2}^{2}(t)-\widetilde{A}_{3}^{2}(t)-\widetilde{A}_{4}^{2}(t) \\
& <0 .
\end{aligned}
$$

Since the Lyapunov function $V\left(e_{1}, e_{2}, e_{3}, e_{4}, \widetilde{a}, \widetilde{b}, \widetilde{c}, \widetilde{d}\right)$ is positive definite and its derivative $\dot{V}\left(e_{1}, e_{2}, e_{3}, e_{4}, \widetilde{a}, \widetilde{b}, \widetilde{c}, \widetilde{d}\right)$ is negative definite in the neighborhood of the zero solutions for (12a) and (12b), according to the Lyapunov stability theory, the zero solutions of error states dynamic and parameters error vector are asymptotically stable; namely, the slave system equation (11) can asymptotically converge to its master system equation (10) with the adaptive control law equation (36) and the estimation parameter update law equation (37). The generalized adaptive synchronization with uncertain parameters concept proof had to be completed. The numerical simulation results are shown in Figures 8, 9, 10, and 11.

\section{Conclusion}

A generalized adaptive synchronization with uncertain chaotic parameters is new chaos synchronization concept.
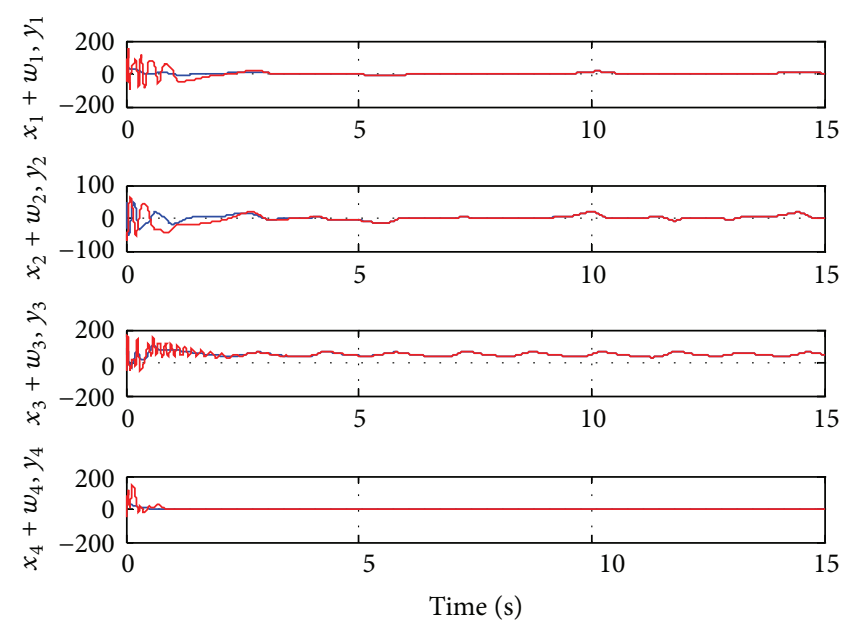

$x+w$

Slave

FIGURE 8: Time histories of the generalized signal (master $x$ system plus given function $w$ ) and slave system for Case III.
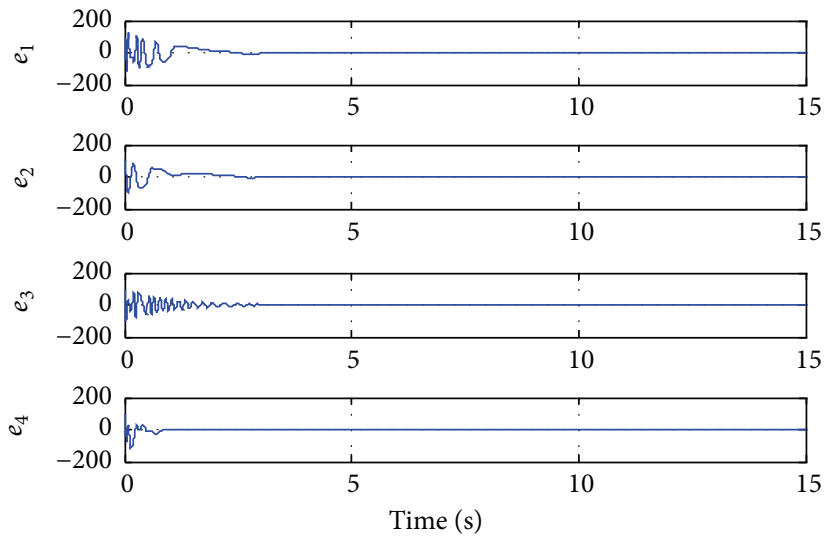

FIGURE 9: Time histories of error states for Case III.

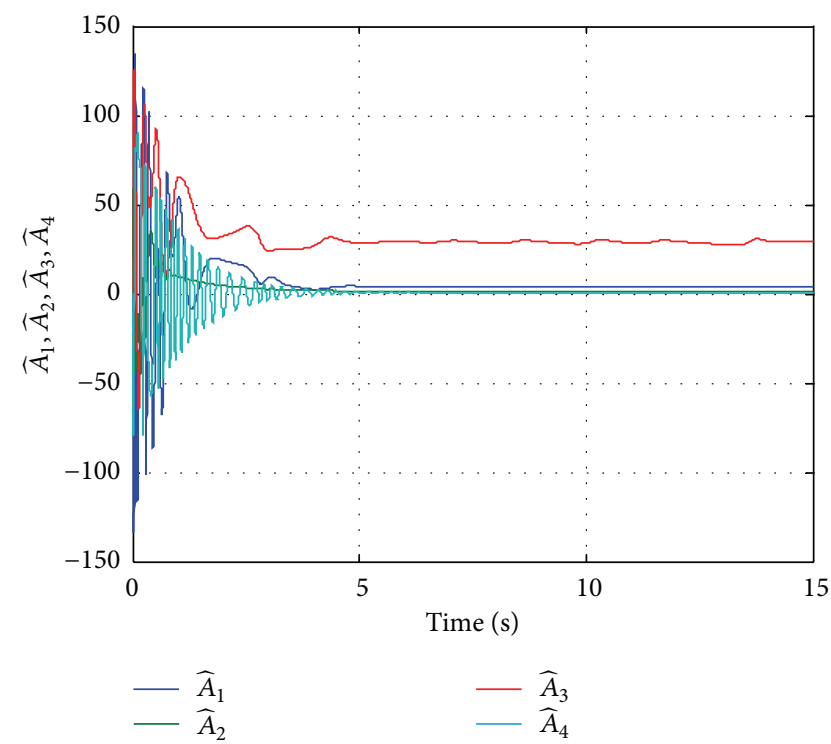

FIgURE 10: Time histories of estimated parameters for Case III. 


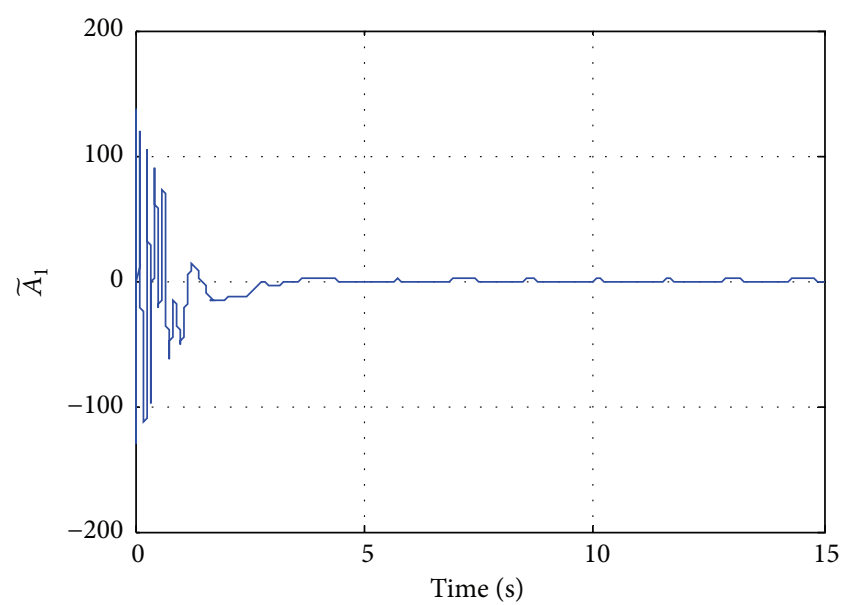

(a)

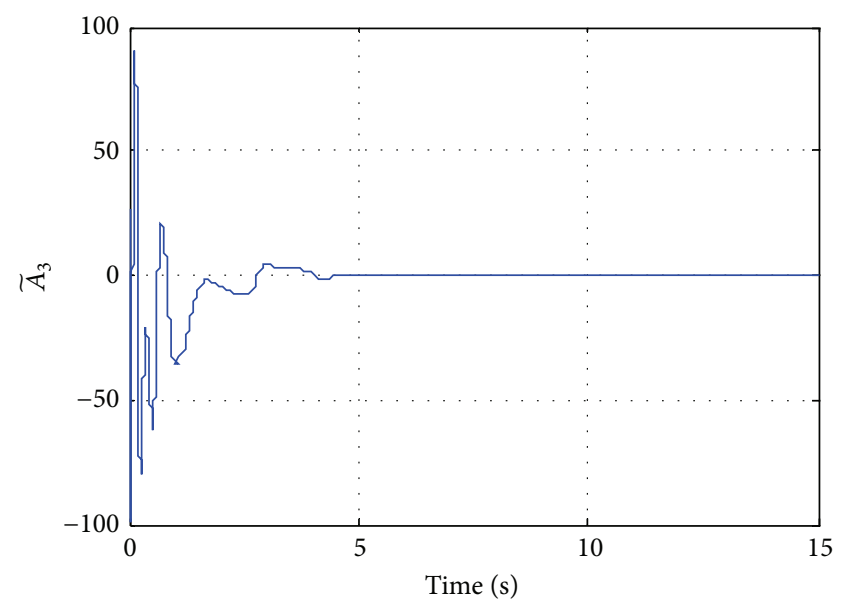

(c)

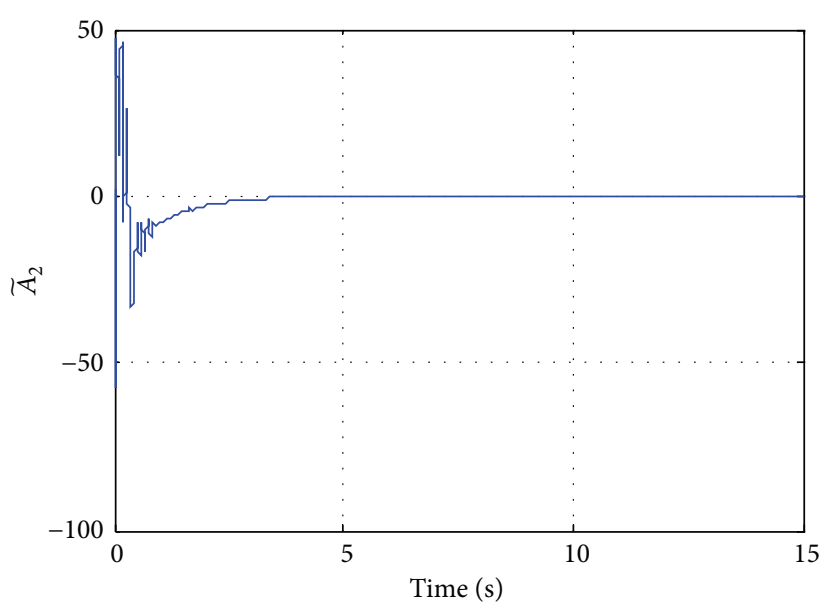

(b)

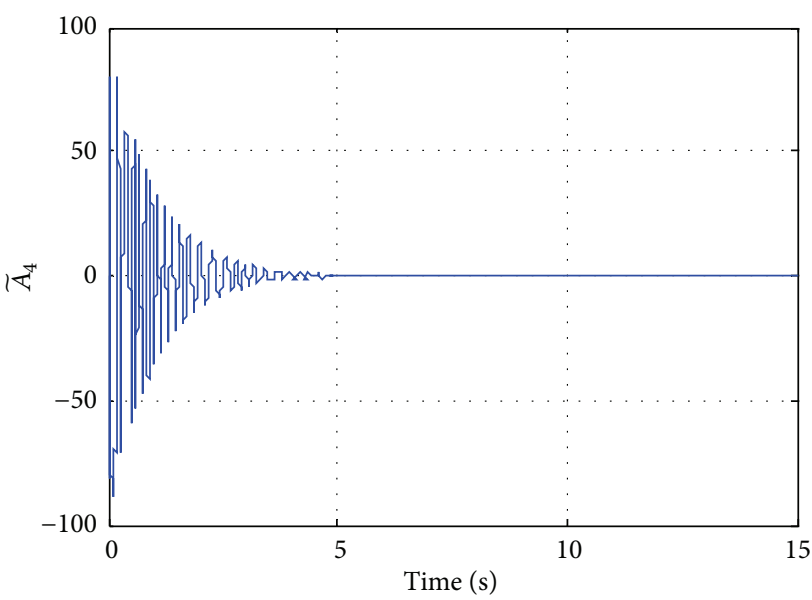

(d)

FIgURE 11: Time histories of different parameters for Case III.

The theoretical analysis and numerical simulation results of three cases, adaptive synchronization, generalized adaptive synchronization, and generalized adaptive synchronization with uncertain parameters, are shown in the corresponding figures which imply that the adaptive controllers and update laws we designed are feasible and effective. In this paper, the three examples can be used to increase the security of secret communication system.

\section{Conflict of Interests}

The authors declare that there is no conflict of interests regarding the publication of this paper.

\section{Acknowledgment}

This research was supported by the National Science Council, China, under Grant no. NSC 102-2221-E-011-034.

\section{References}

[1] L. M. Pecora and T. L. Carroll, "Synchronization in chaotic systems," Physical Review Letters, vol. 64, no. 8, pp. 821-824, 1990.
[2] J. R. Terry and G. D. Vanwiggeren, "Chaotic communication using generalized synchronization," Chaos, Solitons and Fractals, vol. 12, no. 1, pp. 145-152, 2001.

[3] W. Guo Xu and Q. Shu Li, "Chemical chaotic schemes derived from NSG system," Chaos, Solitons and Fractals, vol. 15, no. 4, pp. 663-671, 2003.

[4] Z.-M. Ge and C.-H. Yang, "Symplectic synchronization of different chaotic systems," Chaos, Solitons and Fractals, vol. 40, no. 5, pp. 2532-2543, 2009.

[5] Z.-M. Ge and C.-H. Yang, "The generalized synchronization of a Quantum-CNN chaotic oscillator with different order systems," Chaos, Solitons and Fractals, vol. 35, no. 5, pp. 980-990, 2008.

[6] Z.-M. Ge and C.-H. Yang, "Synchronization of complex chaotic systems in series expansion form," Chaos, Solitons \& Fractals, vol. 34, no. 5, pp. 1649-1658, 2007.

[7] U. E. Vincent, "Synchronization of identical and non-identical 4-D chaotic systems using active control," Chaos, Solitons and Fractals, vol. 37, no. 4, pp. 1065-1075, 2008.

[8] L. Stenflo, "Generalized Lorenz equations for acoustic-gravity waves in the atmosphere," Physica Scripta, vol. 53, no. 1, pp. 8384, 1996. 
[9] C.-H. Yang, "Enhanced symplectic synchronization between two different complex chaotic systems with uncertain parameters," Abstract and Applied Analysis, vol. 2013, Article ID 193138, 12 pages, 2013.

[10] S.-Y. Li, C.-H. Yang, and C.-T. Lin, "Chaotic motions in the real fuzzy electronic circuits," Abstract and Applied Analysis, vol. 2013, Article ID 875965, 8 pages, 2013.

[11] C.-H. Yang, "Symplectic synchronization of Lorenz-Stenflo system with uncertain chaotic parameters via adaptive control," Abstract and Applied Analysis, vol. 2013, Article ID 528325, 14 pages, 2013.

[12] S.-Y. Li, C.-H. Yang, L.-W. Ko, C.-T. Lin, and Z.-M. Ge, "Implementation on electronic circuits and RTR pragmatical adaptive synchronization: time-reversed uncertain dynamical systems' analysis and applications," Abstract and Applied Analysis, vol. 2013, Article ID 909721, 10 pages, 2013.

[13] C.-H. Yang, "Chaos hybrid generalized synchronization of liuchen system by GYC partial region stability theory," Journal of Computational and Theoretical Nanoscience, vol. 10, no. 4, pp. 825-831, 2013.

[14] C. H. Yang, P. C. Tsen, S. Y. Li, and Z. M. Ge, "Pragmatical adaptive synchronization by variable strength linear coupling," Journal of Computational and Theoretical Nanoscience, vol. 10, no. 4, pp. 1007-1013, 2013.

[15] C.-H. Yang, "Chaos control of the Quantum-CNN systems," Journal of Computational and Theoretical Nanoscience, vol. 10, no. 1, pp. 171-176, 2013.

[16] Z. Liu, "The first integrals of nonlinear acoustic gravity wave equations," Physica Scripta, vol. 61, no. 5, article 526, 2000.

[17] C. Zhou, C. H. Lai, and M. Y. Yu, "Bifurcation behavior of the generalized Lorenz equations at large rotation numbers," Journal of Mathematical Physics, vol. 38, no. 10, pp. 5225-5239, 1997.

[18] S. Banerjee, P. Saha, and A. R. Chowdhury, "Chaotic scenario in the Stenflo equations," Physica Scripta, vol. 63, no. 3, pp. 177-180, 2001.

[19] Y. Chen, X. Wu, and Z. Gui, "Global synchronization criteria for two Lorenz-Stenflo systems via single-variable substitution control," Nonlinear Dynamics, vol. 62, no. 1-2, pp. 361-369, 2010.

[20] L. Yang and J. Jiang, "Adaptive synchronization of driveresponse fractional-order complex dynamical networks with uncertain parameters," Communications in Nonlinear Science and Numerical Simulation, vol. 19, no. 5, pp. 1496-1506, 2014.

[21] D. Zhu, C. Liu, and B. Yan, "Modeling and adaptive pinning synchronization control for a chaotic-motion motor in complex network," Physics Letters A, vol. 378, no. 5-6, pp. 514-518, 2014.

[22] W. Xiao-Qun and L. Jun-An, "Parameter identification and backstepping control of uncertain Lü system," Chaos, Solitons and Fractals, vol. 18, no. 4, pp. 721-729, 2003.

[23] Y. Yu and S. Zhang, "Controlling uncertain Lü system using backstepping design," Chaos, Solitons and Fractals, vol. 15, no. 5, pp. 897-902, 2003.

[24] J. A. Laoye, U. E. Vincent, and S. O. Kareem, "Chaos control of $4 \mathrm{D}$ chaotic systems using recursive backstepping nonlinear controller," Chaos, Solitons and Fractals, vol. 39, no. 1, pp. 356$362,2009$.

[25] M. T. Yassen, "Chaos control of chaotic dynamical systems using backstepping design," Chaos, Solitons \& Fractals, vol. 27, no. 2, pp. 537-548, 2006.

[26] W. M. Bessa, A. S. de Paula, and M. A. Savi, "Chaos control using an adaptive fuzzy sliding mode controller with application to a nonlinear pendulum," Chaos, Solitons and Fractals, vol. 42, no. 2, pp. 784-791, 2009.

[27] J.-F. Chang, M.-L. Hung, Y.-S. Yang, T.-L. Liao, and J.-J. Yan, "Controlling chaos of the family of Rössler systems using sliding mode control," Chaos, Solitons \& Fractals, vol. 37, no. 2, pp. 609622,2008 .

[28] S. Zhankui and K. Sun, "Nonlinear and chaos control of a microelectro-mechanical system by using second-order fast terminal sliding mode control," Communications in Nonlinear Science and Numerical Simulation, vol. 18, no. 9, pp. 2540-2548, 2013.

[29] D. Chen, W. Zhao, J. C. Sprott, and X. Ma, "Application of Takagi-Sugeno fuzzy model to a class of chaotic synchronization and anti-synchronization," Nonlinear Dynamics, vol. 73, no. 3, pp. 1495-1505, 2013.

[30] Y. Nian and Y. Zheng, "Controlling discrete time T-S fuzzy chaotic systems via adaptive adjustment," Physics Procedia, vol. 24, pp. 1915-1921, 2012.

[31] H. Hua, Y. Liu, J. Lu, and J. Zhu, "A new impulsive synchronization criterion for T-S fuzzy model and its applications," Applied Mathematical Modelling, vol. 37, pp. 8826-8835, 2013.

[32] S. Yanchuka and T. Kapitaniak, "Chaos-hyperchaos transition in coupled Rössler systems," Physics Letters A, vol. 290, pp. 139144, 2001.

[33] P. Perlikowski, A. Stefanski, and T. Kapitaniak, "Ragged synchronizability and clustering in a network of coupled oscillators," in Advances in Nonlinear Dynamics and Synchronization, pp. 49-75, 2009.

[34] M. Chadlia, A. Abdob, and S. X. Dingb, " $\mathrm{H}_{-} / \mathrm{H}_{\infty}$ fault detection filter design for discrete-time Takagi-Sugeno fuzzy system," Automatica, vol. 49, no. 7, pp. 1996-2005, 2013.

[35] M. Chadli and H. R. Karimi, "Robust observer design for unknown inputs takagi-sugeno models," IEEE Transactions on Fuzzy Systems, vol. 21, no. 1, pp. 158-164, 2013.

[36] S. Aouaouda, M. Chadli, V. Cocquempot, and M. Tarek Khadir, "Multi-objective $H_{-} H_{\infty}$ fault detection observer design for Takagi-Sugeno fuzzy systems with unmeasurable premise variables: descriptor approach," International Journal of Adaptive Control and Signal Processing, vol. 27, no. 12, pp. 1031-1047, 2013.

[37] M. Chadlia, I. Zelinkab, and T. Youssef, "Unknown inputs observer design for fuzzy systems with application to chaotic system reconstruction," Computers \& Mathematics with Applications, vol. 66, no. 2, pp. 147-154, 2013.

[38] I. Zelinka, M. Chadli, D. Davendra, R. Senkerik, and R. Jasek, "An investigation on evolutionary reconstruction of continuous chaotic systems," Mathematical and Computer Modelling, vol. 57, no. 1-2, pp. 2-15, 2013.

[39] C.-H. Yang and C.-L. Wu, "Nonlinear dynamic analysis and synchronization of four-dimensional Lorenz-Stenflo system and its circuit experimental implementation," Abstract and Applied Analysis, vol. 2014, Article ID 213694, 17 pages, 2014. 

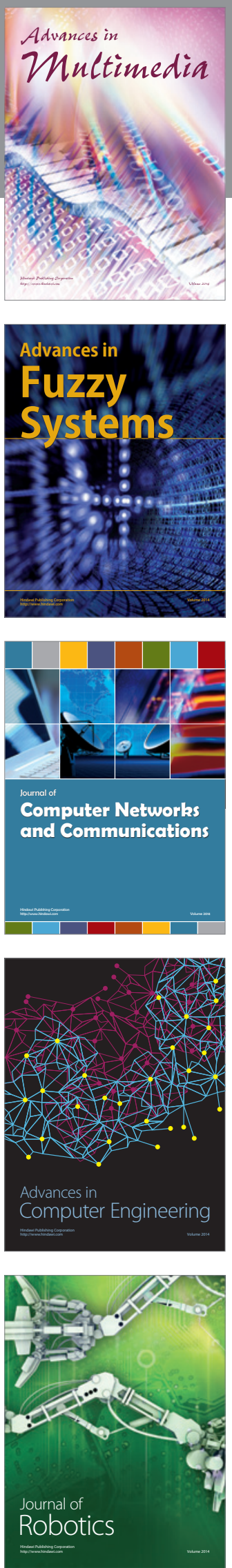

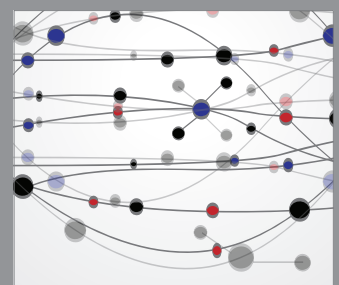

The Scientific World Journal
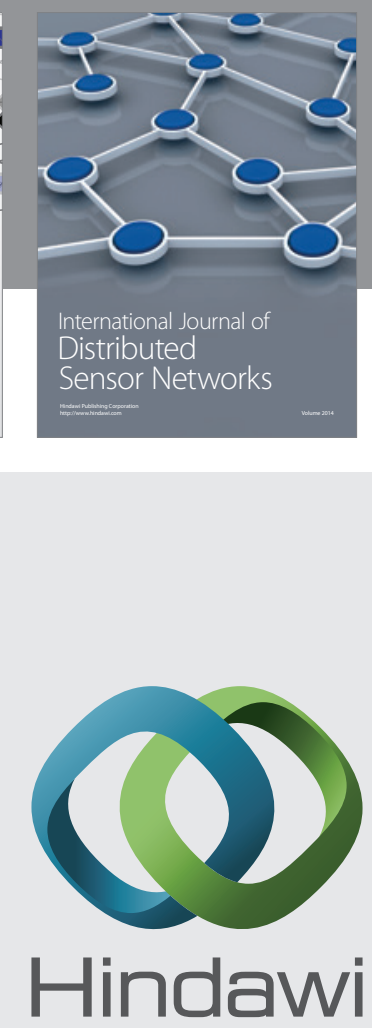

Submit your manuscripts at

http://www.hindawi.com
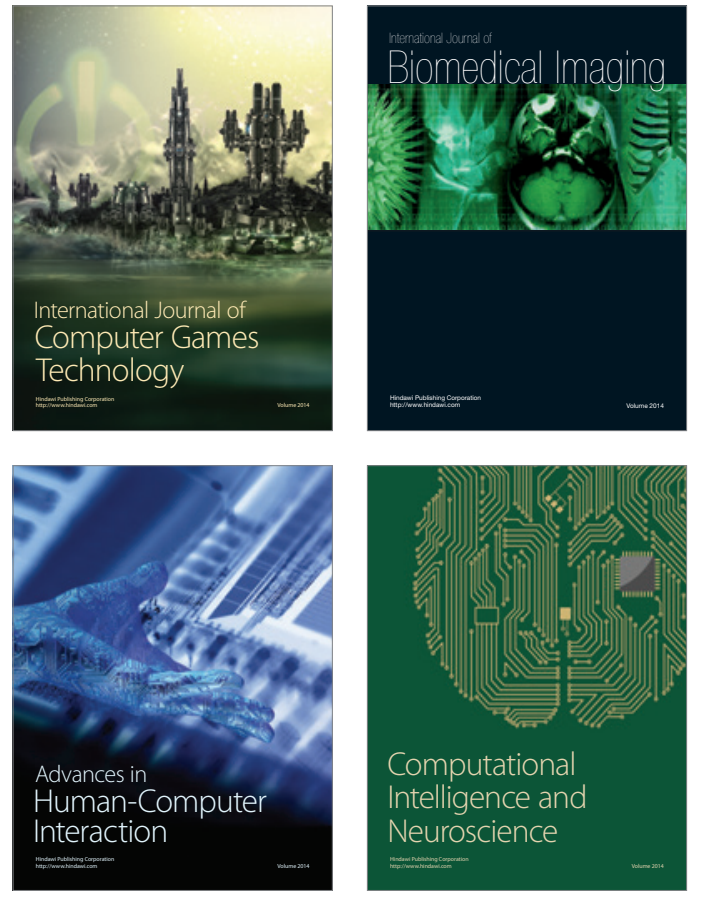
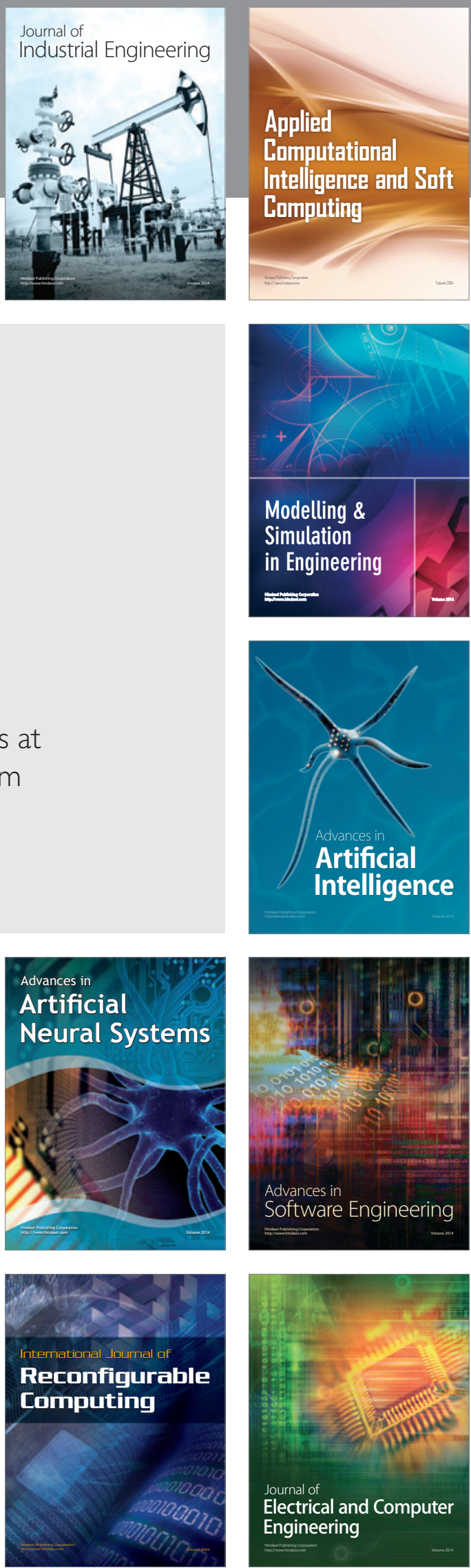